\title{
Low-Frequency Fatigue as an Indicator of Eccentric Exercise-Induced Muscle Injury: The Role of Vitamin E
}

\author{
Antonios Kyparos, ${ }^{1}$ Michalis G. Nikolaidis, ${ }^{1}$ Konstantina Dipla, ${ }^{1}$ Andreas Zafeiridis, ${ }^{1}$ \\ Vassilis Paschalis, ${ }^{2}$ Gerasimos V. Grivas, ${ }^{1}$ Anastasios A. Theodorou, ${ }^{3}$ Maria Albani, ${ }^{4}$ \\ Chrysoula Matziari, ${ }^{5}$ and Ioannis S. Vrabas ${ }^{1}$
}

\author{
${ }^{1}$ Exercise Physiology and Biochemistry Laboratory, Department of Physical Education and Sports Science at Serres, \\ Aristotle University of Thessaloniki, 62110 Serres, Greece \\ ${ }^{2}$ Department of Physical Education and Sport Science, University of Thessaly, Karies, 42100 Trikala, Greece \\ ${ }^{3}$ Laboratory of Exercise, Health and Human Performance, Research Center, European University of Cyprus, 2404 Nicosia, Cyprus \\ ${ }^{4}$ Laboratory of Physiology, Faculty of Medicine, Aristotle University of Thessaloniki, 54124 Thessaloniki, Greece \\ ${ }^{5}$ Laboratory of Physiology, Department of Physical Education and Sports Science, Aristotle University of Thessaloniki, \\ 54124 Thessaloniki, Greece
}

Correspondence should be addressed to Antonios Kyparos, akyparos@phed-sr.auth.gr

Received 7 April 2012; Accepted 14 June 2012

Academic Editor: Chad M. Kerksick

Copyright ( 2012 Antonios Kyparos et al. This is an open access article distributed under the Creative Commons Attribution License, which permits unrestricted use, distribution, and reproduction in any medium, provided the original work is properly cited.

This study investigates whether vitamin E can attenuate eccentric exercise-induced soleus muscle injury as indicated by the amelioration of in situ isometric force decline following a low-frequency fatigue protocol (stimulation at $4 \mathrm{~Hz}$ for $5 \mathrm{~min}$ ) and the ability of the muscle to recover 3 min after the termination of the fatigue protocol. Adult male Wistar rats were divided into vitamin E-supplemented or placebo-supplemented groups studied at rest, immediately post-exercise or $48 \mathrm{~h}$ post-exercise. Daily DL- $\alpha$-tocopheryl acetate intraperitoneal injections of $100 \mathrm{mg} / \mathrm{kg}$ body mass for 5 consecutive days prior to exercise doubled its plasma levels. Fatigue index and recovery index expressed as a percentage of the initial tension. FI at $0 \mathrm{~h}$ post- and $48 \mathrm{~h}$ postexercise respectively was $88 \% \pm 4.2 \%$ and $89 \% \pm 6.8 \%$ in the vitamin $\mathrm{E}$ groups versus $76 \% \pm 3 \%$ and $80 \% \pm 11 \%$ in the placebo groups. RI was $99 \% \pm 3.4 \%$ and $100 \% \pm 6 \%$ in the vitamin E groups versus $82 \% \pm 3.1 \%$ and $84 \% \pm 5.9 \%$ in the placebo groups. Complementally to the traditionally recorded maximal force, low-frequency fatigue measures may be beneficial for assessing injury-induced decrease in muscle functionality.

\section{Introduction}

Physical activities involving eccentrically biased muscle contractions have been associated with skeletal muscle injury [1-4] resulting in skeletal muscle functional alterations and performance impairment [5-7]. It has been suggested that a highly reliable and widely used functional index for evaluating eccentric contraction-induced muscle injury in animals is the measurement of the electrically elicited maximal isometric tetanic force $\left(P_{o}\right)$, whereas in humans is the measurement of maximal voluntary contraction torque [8].

Interestingly though, several lines of evidence from human and animal studies have recognized that after eccentric contractions the reduction in muscle force is more profound at submaximal tension elicited by low frequencies of stimulation, rather than maximal tetanic tension elicited by high frequencies of stimulation. Although Westerblad and Allen [9] have proposed the terminology delayed low-frequency recovery to describe the prolonged fatigueinduced weakness at low stimulation frequencies, yet the term widely used to describe this physiological phenomenon is low-frequency fatigue.

In humans, the loss of muscle force was more profound at low-frequency stimulation when muscle was exercised isometrically at a long length [10] or after exercise in which muscle was actively stretched [11]. In animal studies, the force depression at low frequencies of stimulation following 
eccentric contractions has been recorded in single mouse fibers in vitro [5], in rat muscle bundles preparation in vitro [12], as well as in entire rat muscle in vitro [13]. Lowfrequency fatigue is caused by a reduction in intracellular calcium $\left(\mathrm{Ca}^{2+}\right)$ concentration, probably as a consequence of a reduced $\mathrm{Ca}^{2+}$ release from the sarcoplasmic reticulum in the injured muscle fibers during the excitation-contraction (E-C) coupling [14-19].

Vitamin $\mathrm{E}$ is a nutritional antioxidant widely used to attenuate oxidative stress and muscle injury associated with eccentric exercise [20-22]. The potential protective effect of vitamin $\mathrm{E}$ against exercise-induced muscle injury and disturbance of redox homeostasis is attributed to its properties to act as a potent chain breaking antioxidant [23] and stabilizer of membrane structure [24-26]. Vitamin E, as an integral part of the membrane bilayer, may prevent oxidation of membrane lipids by scavenging free radicals [23] and contribute to maintaining sarcolemma integrity $[24,26]$.

The relatively little data available from human [27-31] and animal [32-34] studies have failed to show consistently any protective effect of vitamin $\mathrm{E}$ on muscle force deficit caused by eccentric contraction-biased exercise. Only in the study of Shafat et al. [35], in which though a combination of vitamins $\mathrm{E}$ and $\mathrm{C}$ was administered, attenuation of the force decline following eccentric exercise in the supplemented group was reported. It is worth mentioning that in all these studies the potential efficacy of vitamin $\mathrm{E}$ treatment against muscle injury was mainly based upon maintaining maximal muscle force, as assessed by maximal voluntary contraction and/or electrically evoked maximal tetanic tension; rarely was a measure of low-frequency stimulation used.

Undoubtedly, the use of maximal force as a functional measure of exercise-induced muscle injury is of great importance, because it reflects the maximal strength that the entire contractile machinery can produce under maximal stimulation. However, maximal tetanic force, which is characterized by prolonged and maximal calcium ion release from the sarcoplasmic reticulum, may not serve as a sensitive indicator of muscle microtrauma as it would be a low-frequency stimulation protocol characterized by transient and limited $\mathrm{Ca}^{2+}$ release. In this regard, stimulation at low frequencies may better reflect $\mathrm{Ca}^{2+}$ kinetics, thus revealing any potential E-C coupling failure induced by eccentric exercise. Moreover, any potential protective effect of vitamin $\mathrm{E}$ or other nutritional intervention on muscle injury should be strong enough for this effect to be statistically shown, whereas weaker, yet true, effects might not be detected.

Indeed, it has been recently demonstrated in vitaminE-supplemented rats that, even though maximal isometric tetanic force $\left(P_{o}\right)$ was not clearly spared following eccentric contraction-biased exercise, single twitch force $\left(P_{t}\right)$, which reflects $\mathrm{Ca}^{2+}$ kinetics in the muscle [36], declined significantly less compared to the placebo conditions [32]. Based on this evidence, we hypothesized that any potential effect of vitamin $\mathrm{E}$ treatment to attenuate muscle injuryinduced force decline could be better tested by utilizing a low-frequency stimulation protocol. The implementation of a low-frequency fatigue protocol may be a sensitive screening method for detecting alterations in muscle function associated with microdamage that, otherwise, could not be detected under conditions of maximal stimulation.

If this is the case, then, along with the regularly used $P_{o}$ measure, the incorporation of a low-frequency fatigue test as a standard procedure for assessing muscle functionality following eccentric contraction-biased exercise would provide a more comprehensive approach to muscle injury and the effectiveness of the interventions implemented to attenuate the injury. For, unlike maximal force which is rarely used in everyday activities, low-frequency stimulation is commonly used in everyday tasks [37]. In this regard, the force deficit at low frequencies of stimulation may be of even greater physiological and clinical importance than the deficit in maximal force, because the former may compromise functionality in daily activities.

Therefore, the aim of the present study was to explore the possibility that a low-frequency fatigue protocol can clearly detect small alterations in muscle force induced by muscle-damaging exercise. To accomplish this objective, vitamin $\mathrm{E}$, a nutritional compound know to have some prophylactic effects on injury-induced force deficit [32,35], was employed. Vitamin-E-treated or placebo-treated rats ran downhill on a treadmill and studied at rest, immediately postexercise or $48 \mathrm{~h}$ postexercise. Muscle injury was assessed by implementing a very-low-frequency fatigue protocol (stimulation at $4 \mathrm{~Hz}$ for $5 \mathrm{~min}$ ) in the soleus muscle in situ. The ability of the muscle for immediate recovery was also estimated by recording isometric single twitch tension $3 \mathrm{~min}$ after the completion of the fatigue protocol.

\section{Materials and Methods}

2.1. Animals. The project was approved by the institutional review board and the appropriate state authority. All procedures were in accordance with the European Union guidelines for the care and use of laboratory animals, as well as the "Principles of laboratory animal care" (NIH publication No. 86-23, revised 1985). Sixty adult (8 to 10 week-old) male Wistar rats, weighing $220-270$ g, were used in the study. The animals were housed under a $12 \mathrm{~h}$ light: $12 \mathrm{~h}$ dark cycle, controlled temperature $\left(18-21^{\circ} \mathrm{C}\right)$, and controlled humidity (50-70\%). Commercial rat chow (ELVIZ, Plati, Greece) and tap water were provided ad libitum.

Rats were randomly assigned into six groups as follows: placebo-treated sedentary control ( $\mathrm{Pb}$-Sed), placebo-treated and studied immediately postexercise $(\mathrm{Pb}-\mathrm{Ex} 0)$, placebotreated and studied $48 \mathrm{~h}$ postexercise ( $\mathrm{Pb}-\mathrm{Ex} 48)$, vitamin-Etreated sedentary control (VE-Sed), vitamin-E-treated and studied immediately postexercise (VE-Ex0), and vitamin Etreated and studied $48 \mathrm{~h}$ postexercise (VE-Ex48). Experimental design is shown in Figure 1.

We examined muscle injury at 0 and $48 \mathrm{~h}$ postexercise because these time points highlight the two main stages (i.e., initial/autogenetic and phagocytic) of the four-stage muscle injury process, as proposed by Armstrong [38]. The 


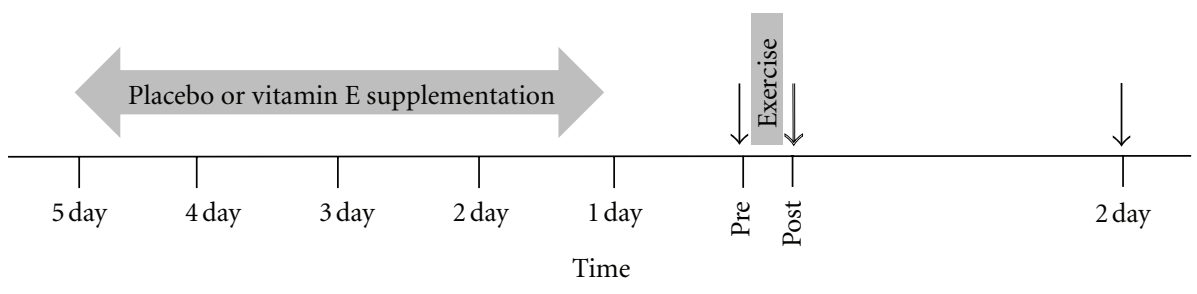

FIGURE 1: Experimental design. Rats were injected intraperitoneally with either placebo (grain oil) or vitamin $\mathrm{E}$ ( $100 \mathrm{mg} / \mathrm{kg}$ body mass of DL- $\alpha$-tocopheryl acetate per day) for 5 consecutive days prior to examination (placebo-treated and vitamin-E-treated sedentary control groups) or exercise (placebo-treated and vitamin-E-treated exercised groups studied immediately post and 2 days post exercise), respectively. Downward arrows indicate the time of assessment.

initial/autogenetic stage reflects primarily the initiation of degrading cellular structures via $\mathrm{Ca}^{2+}$-activated proteolytic and lipolytic events initially induced by the mechanical component of eccentric exercise. During the phagocytic stage, which is marked by a typical inflammatory response in the tissue, the injury is mainly attributed to the metabolic events associated with the production of reactive oxygen species, the breakdown of the injured myofibrils, and the removal of fiber debris [38].

2.2. Vitamin E Supplementation. The animals of the vitaminE-treated sedentary control group and those of the vitaminE-treated exercised groups were injected intraperitoneally (i.p.) with $100 \mathrm{mg} / \mathrm{kg}$ body mass of $\mathrm{DL}-\alpha$-tocopheryl acetate per day for 5 consecutive days prior to examination or exercise, respectively [39, 40]. Vitamin E was diluted in grain oil at a concentration of $4 \%(\mathrm{w} / \mathrm{v})$. Accordingly, the placebo-treated sedentary and exercised groups were injected with vehicle. Previous administration of similar vitamin E doses have increased vitamin E concentration in muscle [33] and lung [40] and inhibited lipid peroxidation in the lungs [40] and the liver [39]. Based on the vitamin E content of the rat diet $(100 \mathrm{mg} / \mathrm{kg}$ chow $)$, the estimated daily food consumption of an adult laboratory rat in the size range of 220-270 (10-20 g a day), and the injected daily dose ( $100 \mathrm{mg} / \mathrm{kg}$ body mass), it is estimated that for rats in this size range the daily vitamin $\mathrm{E}$ intake was $22-27 \mathrm{mg}$ for vitaminE-treated rats and 1-2 mg for placebo-treated rats. This is approximately 15-20 times the vitamin E intake through the normal diet.

2.3. Exercise Protocol. Exercise was performed after an overnight fast and 16-18 h after the last injection. The exercise protocol consisted of $90 \mathrm{~min}$ of intermittent downhill running on a motor-driven treadmill. After a few minutes of familiarization on the treadmill, the rats performed 18 bouts of 5-minute running at a speed of $16 \mathrm{~m} / \mathrm{min}$ down a $16^{\circ}$ inclination, with a 2 minute rest between bouts. After the completion of exercise protocol, the placebo-treated and vitamin-E-treated rats to be studied $48 \mathrm{~h}$ postexercise were placed back in their cages with free access to food and water until they were examined. Previous studies have shown that this exercise protocol induced injury in the soleus and other rat hindlimb muscles $[1,4,41,42]$.
2.4. Plasma Vitamin E Concentration. Blood samples were collected either at rest (16-18 h after the last injection) or immediately after exercise or $48 \mathrm{~h}$ following exercise as per group as follows. Under deep anaesthesia with chloral hydrate $(4.5 \%, 1 \mathrm{~mL} / 100 \mathrm{~g}$ body mass i.p.), about $1 \mathrm{~mL}$ of blood was drawn from the right jugular vein using a heparin-treated syringe. Blood was then centrifuged at $3000 \times \mathrm{g}$ for $10 \mathrm{~min}$ and the resulting plasma was collected in eppendorf vials, snap frozen in liquid nitrogen and stored at $-80^{\circ} \mathrm{C}$ until analysis. In 3 animals from each group, plasma vitamin $\mathrm{E}$ was determined using high-performance liquid chromatography (Jasco HPLC System, Great Dunmow, UK) according to Nierenberg and Nann [43]. Each assay was run in duplicate and the interassay coefficient of variation (CV) was less than $10 \%$.

2.5. In Situ Isometric Tension Recording. After blood sampling, in situ isometric tension of the soleus muscle was recorded following a procedure previously described [32]. Under the same anaesthesia as above (chloral hydrate $4.5 \%$, $1 \mathrm{~mL} / 100 \mathrm{~g}$ body mass i.p.), the hair of the lower limbs was shaved up to the knee joint and the area was cleaned lightly with $70 \%$ ethanol. The skin was then sectioned and a longitudinal incision was made on the lateral surface of the right hindlimb over the area covered by gluteus superficialis. Adjacent muscles were gently reflected using blunt-tip forceps to expose the sciatic nerve. Likewise, a small incision was made on the back of the ankle, thus uncovering the distal (Achilles) tendon. The gastrocnemius muscle was retracted carefully to avoid rupturing the blood vessels, and the intact soleus muscle was exposed. The tendon was then tied with $3 / 0$ silk thread and cut distally.

The rat was then placed prone on a stable rodent surgery table and was prepared for tension recording. Steel pins were inserted through the knee and ankle joints to stabilize the hindlimb, and cloth tape was used to secure the foot perpendicularly to the lower leg. Pins were supported with magnetic stand holders throughout the experiment. The tendon of the soleus muscle was attached to a strain-gauge transducer (UFI, Morro Bay, CA) by short silk suture, and bipolar silver electrodes were placed under the sciatic nerve, which was held in a relaxed position. Isometric contractions were evoked by stimulating the sciatic nerve (Digitimer DS9A stimulator, Hertfordshire, UK) using supramaximal $(3-8 \mathrm{~V})$ square pulses of $0.5 \mathrm{~ms}$. Tetanic twitch stimulation 
was set at $350 \mathrm{~ms}$. The signal from the transducer was amplified by a DC amplifier (Neurolog NL 107, Digitimer, Hertfordshire, UK), displayed on an oscilloscope screen (Fluke PM 3380A, Everett, WA), stored in a computer, and processed using data acquisition software (FlukeView combiscope software, Everett, WA). A pulse programmer (Digitimer D4030, Hertfordshire, UK) controlled all devices during tension recording.

The muscle was adjusted to the optimal length $\left(L_{o}\right)$ using a micromanipulator (Prior Scientific, Rockland, MA) allowing motion in all three directions. $L_{o}$ was defined as the muscle length at which maximal twitch tension was obtained. It took 5-6 single twitch trials to set $L_{o}$. Once this was set, a 1-minute resting period was allowed before the actual recordings. Throughout the recordings, the longitudinal axis of the muscle remained aligned to the longitudinal axis of the transducer, and both remained parallel to the tibia.

A very low-frequency fatigue protocol (stimulation at $4 \mathrm{~Hz}$ for $5 \mathrm{~min}$ ) was implemented to assess soleus muscle fatigability as described below. Throughout tension recording, the rat was kept warm with a heating pad. A diffuse heat source was also placed in close proximity to the surgery table. The depth of anaesthesia was assured by the constriction of the pupils as well as simple sensory tests, such as the absence of eye blinking when the eyelid was touched and the absence of foot withdrawal when the foot was pinched. When necessary, anaesthesia was maintained by administering approximately $10 \%$ of the initial dose. The sciatic nerve and soleus muscle were kept moist by periodically sprinkling Krebs solution at $37^{\circ} \mathrm{C}$. Immediately after tension recording, the soleus muscle was excised and weighed on an electronic scale with an accuracy of $0.001 \mathrm{~g}$. Animals were then euthanized by an overdose of chloral hydrate injected intravenously. Normalization of the data from muscles of different sizes was achieved by expressing generated force per muscle mass.

2.6. Fatigue Protocol. A muscle fatigue protocol of very lowfrequency (stimulation at $4 \mathrm{~Hz}$ for $5 \mathrm{~min}$ ) was applied to test for fatigability. The decrease in tension after the $5 \mathrm{~min}$ stimulated contraction period was expressed as a percentage of the initial tension, denoting the fatigue index (FI). To estimate the ability for immediate muscle recovery, isometric single twitch tension $\left(P_{t}\right)$ was recorded 3 minute after the completion of the fatigue protocol. The tension recorded after the $3 \mathrm{~min}$ recovery period was expressed as a percentage of the initial tension, denoting the recovery index (RI). For both FI and RI, an initial tension was considered the greatest force of the first three twitches recorded in the beginning of the $5 \mathrm{~min}$ fatigue protocol. To ensure that the muscle retained its $L_{o}$ after repeated contractions during the $5 \mathrm{~min}$ fatigue protocol, an average of 2-3 single-twitch trials were applied immediately after the 3 minute recovery period. Once $L_{o}$ was confirmed, the actual single twitch was recorded after 1 minute.

2.7. Statistical Analysis. Data were analyzed with the SPSS, version 12, software (SPSS, Chicago, IL) and presented as mean \pm SD. The distribution of all dependent variables was examined by the Shapiro-Wilk test and was found not to differ significantly from normal. To evaluate the potential protective effects of vitamin E supplementation against exercise-induced muscle injury as reflected by force decline during the fatigue test and the immediate recovery rate, we performed $2 \times 3$ (supplementation by time) ANOVA followed by simple main effect analysis. The statistical significance level was set at $\alpha=0.05$. To determine the meaningfulness of the effects of exercise and vitamin $\mathrm{E}$ supplementation on muscle injury markers, effect sizes (ESs) were calculated for FI and RI as the difference between post- and preexercise mean values divided by the standard deviation of the preexercise value. According to the original Cohen's scale, effect size is 0.2 for small, 0.5 for moderate and 0.8 for large effects [44] and according to the modified Cohen's scale, effect size is 0.2 for small, 0.6 for moderate, 1.2 for large, 2.0 for very large, and 4.0 for nearly perfect effects (http://sportsci.org/resource/stats/). Due to the prepost characteristic of our intervention and an improved response in fact being a reduction in the post value, the ES inherently will be negative. To avoid confusion in the interpretation of the calculated ES, we decided to present ES as absolute values.

\section{Results}

3.1. Plasma Vitamin E Concentration. The data referring to vitamin $\mathrm{E}$ concentration in plasma have been initially presented in a recently published paper of a relative work from our group [32]. Because of the small sample size used for the particular analysis ( $n=3$ per group), only descriptive statistics were applied on these data. No further inferential statistical comparison was performed on these values. Vitamin E administration doubled the concentration of vitamin $\mathrm{E}$ in plasma in the sedentary control group (VESed group: $18.5 \mathrm{mg} / \mathrm{L} \pm 3.3 \mathrm{mg} / \mathrm{L}$, range $15.9-22.2 \mathrm{mg} / \mathrm{L}$ versus $\mathrm{Pb}$-Sed: $9.3 \mathrm{mg} / \mathrm{L} \pm 0.4 \mathrm{mg} / \mathrm{L}$, range $8.9-9.7 \mathrm{mg} / \mathrm{L}$ ). Furthermore, the vitamin-E-treated exercised rats had consistently higher vitamin E plasma levels compared to that of the placebo-treated exercised counterparts, both immediately postexercise (VE-Ex0: $12.3 \pm 0.7 \mathrm{mg} / \mathrm{L}$, range $11.8-13.1 \mathrm{mg} / \mathrm{L}$ versus $\mathrm{Pb}-\mathrm{Ex} 0: 7.4 \pm 2.3 \mathrm{mg} / \mathrm{L}$, range $5.9-$ $10.1 \mathrm{mg} / \mathrm{L}$ ) and, at $48 \mathrm{~h}$ postexercise (VE-Ex48: $13.8 \pm$ 1.1 , range $13.0-15.0 \mathrm{mg} / \mathrm{L}$ versus $\mathrm{Pb}-\mathrm{Ex} 48: 10.2 \pm 1.3 \mathrm{mg} / \mathrm{L}$, range $8.8-11.3 \mathrm{mg} / \mathrm{L}$ ); yet these vitamin-E concentrations were lower compared to that measured in the vitamin-Etreated sedentary control group.

3.2. Fatigue Index and Recovery Index. Data on soleus muscle fatigability (as reflected by the FI) and ability for immediate recovery (as reflected by the RI) between the placebo-treated exercised groups (i.e., $\mathrm{Pb}-\mathrm{Ex} 0$ and $\mathrm{Pb}-\mathrm{Ex} 48$ ), subject to muscle damaging downhill running (injured muscle), and the placebo-treated sedentary control (Pb-Sed) group (uninjured muscle) have been initially presented elsewhere [45] and now compared with the vitamin Esupplemented condition. Briefly, these data indicated that 
eccentric exercise significantly increased soleus muscle fatigability and decreased the ability for immediate recovery, suggesting that the exercise protocol used in this study induced injury in the soleus muscle.

To examine whether or not vitamin E treatment had any protective effect on muscle injury induced by downhill running, FI and RI were recorded in VE-Ex0 and VEEx48 vitamin-E-treated exercised groups and compared with the values obtained in the $\mathrm{Pb}-\mathrm{Ex} 0$ and $\mathrm{Pb}-\mathrm{Ex} 48$ placebo-treated exercised groups. A marginally nonsignificant supplementation-by-time interaction $(P=0.056)$ and significant main effects of supplementation $(P<0.001)$ and time $(P=0.034)$ in soleus muscle FI were found (Figure 2). In the within groups pairwise comparisons, FI was significantly lower in Pb-Ex0 $(P=0.002)$ and Pb-Ex48 $(P=0.045)$ rats compared to $\mathrm{Pb}$-Sed rats. In the vitamin $\mathrm{E}$ condition, no significant decrease in FI between the exercise groups (VE-Ex0 and VE-Ex48) and the vitamin-E-treated sedentary control group (VE-Sed) was found. In the between groups pairwise comparisons, FI was significantly decreased in the placebo-treated exercised condition compared with the vitamin-E-treated exercised condition, both immediately postexercise $(P<0.001)$ and at $48 \mathrm{~h}$-postexercise $(P=$ $0.009)$. No significant difference $(P>0.05)$ in FI was detected between the $\mathrm{Pb}$-Sed and VE-Sed control groups.

A significant supplementation-by-time interaction $(P<$ $0.001)$, as well as significant main effects of supplementation $(P<0.001)$ and time $(P<0.001)$ in soleus muscle RI were found (Figure 3 ). In the within groups pairwise comparisons, RI was significantly lower in Pb-Ex0 $(P<0.001)$ and $\mathrm{Pb}-$ Ex48 $(P<0.001)$ rats compared to $\mathrm{Pb}$-Sed rats. This was also the case in the vitamin E condition, as a significant decrease in RI between the exercise groups (VE-Ex0 and VE-Ex48) and the vitamin-E-treated sedentary control group (VE-Sed) was found $(P<0.001)$. In the between groups pairwise comparisons, RI was significantly decreased in the placebotreated exercised condition compared with the vitamin Etreated exercised condition, both immediately postexercise $(P<0.001)$ and at $48 \mathrm{~h}$-postexercise $(P=0.009)$. No significant difference $(P>0.05)$ in RI was detected between the $\mathrm{Pb}-\mathrm{Sed}$ and VE-Sed control groups.

\section{Discussion}

This study is part of a larger project aimed at investigating the effects of vitamin E supplementation on muscle injury following downhill running. The objective of the present study, in particular, was to investigate whether a lowfrequency fatigue protocol can clearly detect small alterations in muscle force induced by muscle-damaging exercise. The prophylactic properties of vitamin E against exercise-induced muscle injury, as a potent antioxidant and intramembrane stabilizer alike, was employed for delineating "finest" alterations in muscle force associated with microdamage. Vitamin-E-treated and placebo-treated rats studied at rest, immediately post- or $48 \mathrm{~h}$ postdownhill running. Alternatively, the objective of this study could be formulated as to whether vitamin E can attenuate eccentric exercise-induced

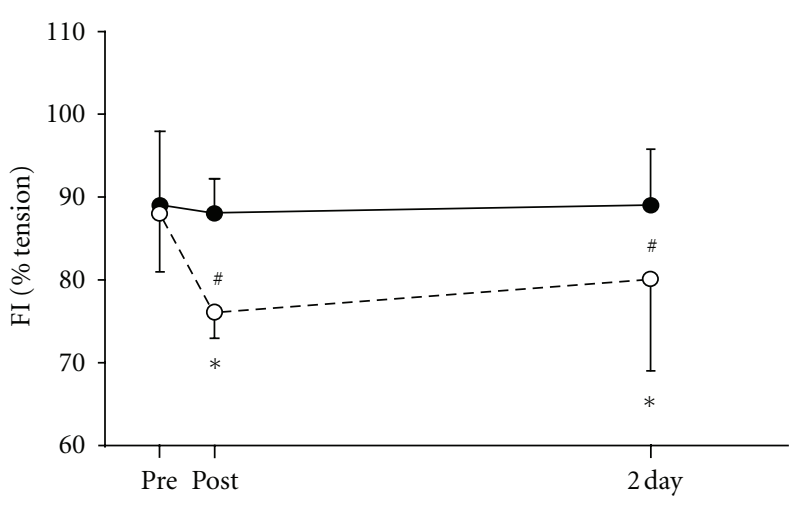

FIgURE 2: Fatigue index (expressed as a percentage of the initial tension) after the implementation of a low-frequency fatigue protocol (stimulation at $4 \mathrm{~Hz}$ for $5 \mathrm{~min}$ ) of the soleus muscle in situ in the placebo-treated (dashed line and $\bigcirc$ ) and vitamin Etreated (solid line and $\bullet$ ) rats (mean and SD, $n=10$ per group). * Significantly different from pre exercise values within the same group $(P<0.05)$. "Significantly different between placebo and vitamin $\mathrm{E}$ at the same time point $(P<0.01)$.

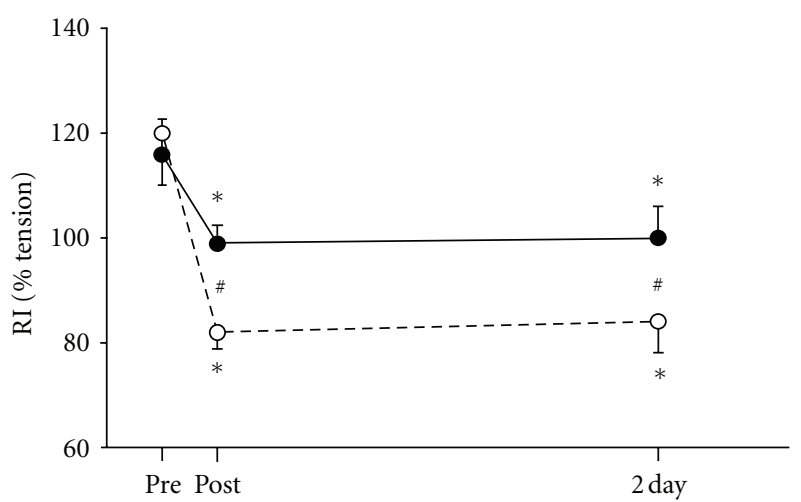

Figure 3: Recovery index (expressed as a percentage of the initial tension) 3 min following the termination of low-frequency fatigue protocol of the soleus muscle in situ in the placebo-treated (dashed line and $\bigcirc$ ) and vitamin-E-treated (solid line and $\bullet$ ) rats (mean and SD, $n=10$ per group). * Significantly different from pre exercise values within the same group $(P<0.001)$. "Significantly different between placebo and vitamin $\mathrm{E}$ at the same time point $(P<0.001)$.

muscle injury as assessed by the amelioration of force decline following the implementation of a low-frequency fatigue protocol and the ability of the muscle for immediate recovery 3 min after the completion of the fatigue protocol.

Our data demonstrate that downhill running significantly increased soleus muscle fatigability and decreased the ability for immediate recovery. The fatigability after the lowfrequency fatigue protocol was more pronounced in the exercised rats immediately postexercise $(\mathrm{Pb}-\mathrm{Ex} 0: 76 \% \pm 3 \%)$ and at $48 \mathrm{~h}$-postexercise (Pb-Ex48: 80\% $\pm 11 \%$ ) compared to the sedentary rats ( $\mathrm{Pb}-\mathrm{Sed}: 88 \% \pm 7 \%)$. The effect sizes for FI in the placebo groups at $0 \mathrm{~h}$ and $48 \mathrm{~h}$ were 1.71 and 1.14, respectively (ES are presented as absolute values). Likewise, the ability of the soleus muscle to recover was diminished in the exercised rats immediately postexercise 
(Pb-Ex0: $82 \% \pm 3.1 \%$ and at 48 h-postexercise (Pb-Ex48: $84 \% \pm 5.9 \%$ ), whereas the RI in the sedentary control group was surprisingly increased by $20 \%$ of the initial single twitch value (Pb-Sed: $120 \% \pm 9.9 \%)$. The effect sizes for RI in the placebo groups at $0 \mathrm{~h}$ and $48 \mathrm{~h}$ were 3.83 and 3.63, respectively. This increase in the RI of the sedentary rats beyond the initial single twitch value was attributed to a staircase effect (treppe phenomenon) that could reflect an increased $\mathrm{Ca}^{2+}$ availability in the uninjured soleus muscle. It is also a criterion of the viability of the preparation.

It appears that the low-frequency protocol-induced minimal fatigue in the nonexercised (uninjured) group, which was fully restored $3 \mathrm{~min}$ after fatigue protocol, suggesting that the force decline in the exercised (injured) groups did not have a metabolic etiology but rather it can be attributed to muscle damage alone. These findings indicate that the exercise protocol used in this study induced injury in the soleus muscle, as evidenced by the significant reduction in the ability of the placebo-treated eccentrically exercised animals (injured muscles) to maintain tension at a lowfrequency fatigue protocol as well as the reduced ability to recover $3 \mathrm{~min}$ after the termination of the fatigue protocol, compared with the sedentary control animals (uninjured muscle).

Vitamin E treatment decreased soleus muscle fatigability and preserved the ability for immediate recovery following downhill running. In the vitamin-E-treated rats, postexercise soleus muscle fatigability (VE-Ex0: $88 \% \pm 4.2 \%$ and VEEx48: $89 \% \pm 6.8 \%)$ was not different compared to the sedentary conditions (VE-Sed: $89 \% \pm 9.9 \%$ ). The effect sizes for FI in the vitamin E groups at $0 \mathrm{~h}$ and $48 \mathrm{~h}$ were 0.11 and 0 , respectively. In addition, as was the case in the placebotreated sedentary group, the RI in the vitamin-E-treated sedentary group was also increased by $16 \%$ of the initial single twitch value (VE-Sed: $116 \% \pm 6.7 \%$ ). Nevertheless, the ability for immediate recovery was essentially preserved in the vitamin-E-treated exercised rats (VE-Ex0: $99 \% \pm 3.4 \%$ and VE-Ex48: $100 \% \pm 6.0 \%)$. The effect sizes for RI in the vitamin $\mathrm{E}$ groups at $0 \mathrm{~h}$ and $48 \mathrm{~h}$ were 2.54 and 2.39, respectively.

When the placebo and vitamin conditions were compared at the same time point, the FI was less pronounced in the VE-Ex0 and VE-Ex48 $(88 \% \pm 4.2 \%$ and $89 \% \pm 6.8 \%)$ groups as compared to that observed in the $\mathrm{Pb}-\mathrm{Ex} 0$ and $\mathrm{Pb}$ Ex48 $(76 \% \pm 3 \%$ and $80 \% \pm 11 \%)$ groups. In addition, the RI was improved in the VE-Ex0 and VE-Ex48 (99\% $\pm 3.4 \%$ and $100 \% \pm 6 \%)$ vitamin-E-treated animals as compared to that observed in the Pb-Ex0 and $\mathrm{Pb}-\mathrm{Ex} 48(82 \% \pm 3.1 \%$ and $84 \% \pm 5.9 \%$ ) placebo-treated counterparts. In fact, the ability of the soleus muscle to produce tension was fully restored $3 \mathrm{~min}$ after the termination of the low-frequency fatigue protocol. Our findings clearly indicate that the vitamin E-treatment used in this study protected soleus muscle from the injury normally induced by downhill running. As compared to the placebo-treated groups, the vitamin-E-treated rats had significantly increased ability to maintain tension at low-frequency fatigue as well as to recover 3 minute after the termination of the fatigue protocol.
A functional measure that has been widely used to assess exercise-induced muscle injury is the maximal tetanic tension, which is associated with maximal intracellular $\mathrm{Ca}^{2+}$ concentration. $P_{o}$, although of great importance, may not serve as a sensitive indicator for detecting smaller functional alterations caused by muscle microtrauma or uncovering potential weaker protective effects of nutritional interventions against muscle damage. We suggest that a low-frequency stimulation protocol, characterized by low intracellular $\mathrm{Ca}^{2+}$ concentration, may also be used as a muscle function index complementing $P_{o}$, because the former may be a more suitable measure to distinguish muscle microdamage induced by exercise.

A considerable number of studies have substantiated that, following eccentric muscle contractions, force deficit is more pronounced at low frequency (submaximal) stimulation rather than high-frequency (maximal) stimulation. The relative isometric force reduction of the rat soleus muscle bundles in vitro at 10 min post injury induced by eccentric contractions was significantly greater at the stimulation frequencies of 10 and $20 \mathrm{~Hz}$ compared to higher stimulation frequencies up to $100 \mathrm{~Hz}$ [12]. This is in agreement with previous data showing a greater relative isometric force reduction at low frequencies postinjury in single fibers of the mouse flexor brevis muscle in vitro. More specifically, the force decline at $10 \mathrm{~min}$ postlengthening contractions was greater at the stimulation frequencies of 30 and $50 \mathrm{~Hz}$ compared to higher stimulation frequencies up to $100 \mathrm{~Hz}$ [5]. In addition, McCully and Faulkner [13] have demonstrated that the isometric force decline of the rat extensor digitorum longus muscle in vitro after lengthening contraction-induced muscle injury was greater at the stimulation frequencies of 100 and $200 \mathrm{~Hz}$ compared to maximal isometric force occurred at $300 \mathrm{~Hz}$. In support to these findings, Jones et al. [10] have also shown that after eccentric contractions the decline in muscle force of the quadriceps and forearm flexors was greater at the electrically evoked low-frequency fatigue $(20: 100 \mathrm{~Hz}$ ratio) compared to maximal voluntary isometric superimposed contraction. In view of this, and the very slow recovery of low-frequency fatigue, the authors suggested that the force decline may be attributed to some form of damage caused by the generation of high forces during lengthening contractions [10]. In all aforementioned studies the force deficit was directly or indirectly attributed to the E-C coupling impairment.

With respect to the effects of vitamin E on muscle force preservation at low frequencies of stimulation following muscle damaging exercise, it has been shown that the subjects treated with a mixture of vitamins $\mathrm{E}$ and $\mathrm{C}$ had smaller decline in electrically evoked force at frequencies of 20 and $50 \mathrm{~Hz}$ immediately post- and at days 1 and 2 postmaximal eccentric contractions of the knee extensor muscles, compared to the placebo controls [35]. In addition, we have recently demonstrated that soleus muscle single twitch force declined significantly less in vitamin-E-treated rats compared to the placebo controls immediately post-downhill running, even though $P_{o}$ was not clearly protected in the vitamin-E-supplemented rats [32]. These findings are in line with previous data on muscle force following downhill 
running in ovariectomized rats treated with estrogen, a compound known to have antioxidant properties similar to vitamin E. More specifically, compared to the placebotreated, the estradiol-treated ovariectomized rats had smaller decline in soleus muscle force at low stimulation frequencies of $10-40 \mathrm{~Hz}$ and less fatigability was recorded immediately post and 3 days post exercise, despite that $P_{o}$ was equally decreased in both treatment conditions [41].

There is overwhelming evidence to support that the main mechanism involved in force decline following eccentric contraction is the E-C coupling failure affecting $\mathrm{Ca}^{2+}$ kinetics $[5,17,46-48]$. The mechanism of $\mathrm{Ca}^{2+}$ kinetics impairment supports our case that low-frequency stimulation might be used as an alternative-or rather complemental to $P_{o}$ functional index of muscle injury induced by eccentric exercise. There is a greater chance that a weaker protective effect of vitamin E against muscle injury is revealed at low stimulation frequencies, whereas this effect may be masked at maximal stimulation. Unlike $P_{o}$, which results from the application of high-frequency stimuli causing prolonged and maximal $\mathrm{Ca}^{2+}$ release from the SR, unfused tetanic force is characterized by a train of low-frequency stimuli challenging the $\mathrm{Ca}^{2+}$ release and $\mathrm{Ca}^{2+}$ reuptake process by the SR. The lower the stimulation frequency, the more transient and limited the $\mathrm{Ca}^{2+}$ release by the SR. In $P_{t}$, for example, the SR $\mathrm{Ca}^{2+}$ channels open abruptly with depolarization and then close rapidly once the membrane is repolarized, thus allowing a very short time for $\mathrm{Ca}^{2+}$ release [36]. In this context, the maximal stimulation of the E-C coupling system may compensate for any potential impairment in SR Ca ${ }^{2+}$ kinetics, and therefore, may mask any functional/force decrements that would normally be manifested under submaximal E-C coupling stimulation. Hence, we proposed that any impairment of $\mathrm{Ca}^{2+}$ kinetics, which was thought to reflect microinjury, is more easily detected via the recordings of single twitch tension and unfused tetanic force at very low stimulation frequencies (e.g., 5 to $30 \mathrm{~Hz}$ ). These measures can be either assessed alone or in a train of stimuli incorporated in a lowfrequency fatigue protocol. For example, a low-frequency fatigue protocol of $10-20 \mathrm{~Hz}$ and duration of 5-10 min might be a very suitable functional tool to detect the microtrauma induced by eccentric-biased exercise.

A limitation of the present study was that plasma vitamin E concentration was determined only in a small number of samples, thus not allowing further inferential statistical comparisons rather than descriptive statistics on these data. Another limitation of the present study was that we did not measure redox homeostasis and oxidative stress biomarkers which could have provided an estimate of the potential contribution of reactive species to low-frequency fatigue as well as whether vitamin E maintained redox homeostasis and alleviated oxidative stress. It has been proposed that the long lasting effects of low-frequency fatigue can be in part attributed to increased generation of reactive species causing structural alterations of the proteins involved in the E-C coupling process, thus affecting $\mathrm{Ca}^{2+}$ kinetics and myofibrillar $\mathrm{Ca}^{2+}$ sensitivity [49-51]. However, data from skinned and intact fibers suggest that the increased sensitivity of the SR
$\mathrm{Ca}^{2+}$ release channels to reactive species is not necessarily followed by a respective physiological activation generated by an action potential $[50,52,53]$. Nevertheless, there is evidence from animal and human studies that support the role of vitamin $\mathrm{E}$ in reducing oxidative stress induced by eccentric exercise. Vitamin E supplementation decreased the susceptibility of the rat soleus muscle homogenates to oxidative stress [34], attenuated the increased protein carbonyls in rat soleus and vastus intermedius muscles [54], and reduced human muscle conjugated denies and urinary thiobarbituric acid reactive substances [55] following downhill running. An interesting approach to look into the role of reactive species in muscle force generation and the potential effectiveness of antioxidants against muscle injury-induced force decline would be to examine the issue within a hormetic framework [56].

In conclusion, our findings suggest that vitamin $\mathrm{E}$ supplementation at the doses and duration administered resulted in a two-fold increase of its concentration in plasma. This dose was effective to protect soleus muscle from injury induced by eccentric contraction-biased exercise as indicated by the decreased fatigability at a low-frequency of stimulation and the almost complete recovery of single-twitch force immediately after fatigue in the vitamin $\mathrm{E}$ condition compared to the placebo condition. This protective effect of vitamin $\mathrm{E}$ at low-frequency fatigue is considered critical and physiologically significant given that low-frequency firing of the neuromuscular system is common in daily activities and, hence, force deficit at these frequencies may possibly compromise everyday functionality. This is not contradictory to the statement we have made elsewhere [32] reporting no physiologically significant protective effects of vitamin $\mathrm{E}$ against eccentric exercise-induced muscle injury. In that study, the physiological significance with respect to muscle injury attenuation was defined merely on the basis of the $P_{o}$. The criterion was the difference in $P_{\mathrm{o}}$ between the vitamin $\mathrm{E}$ and the placebo conditions, only to show the inadequacy to comprehensively evaluate eccentric exerciseinduced force deficit when $P_{o}$ alone is used. For a comprehensive assessment of injury-induced decrease in muscle functionality, it may be beneficial that low-frequency fatigue measures, complementally to the traditionally recorded $P_{o}$, are also determined.

\section{Conflict of Interests}

The authors declare no conflict of interests.

\section{Acknowledgments}

This work was partially funded by a grant award to A. Kyparos by the Research Committee (Research Dissemination Center) of the Aristotle University of Thesssaloniki, Greece. No other funding has been received. Vitamin E was a generous gift by G. A. Pharmaceuticals S. A. Professor Themistoklis Christides is acknowledged for the valuable methodological and technical advice on vitamin $\mathrm{E}$ administration. The authors are grateful to Professor Nikolaos 
Kokolis, Department of Physiology at the Veterinary School of the Aristotle University of Thessaloniki, for kindly opening his laboratory to the authors to perform a portion of the experiments.

\section{References}

[1] R. B. Armstrong, R. W. Ogilvie, and J. A. Schwane, "Eccentric exercise-induced injury to rat skeletal muscle," Journal of Applied Physiology Respiratory Environmental and Exercise Physiology, vol. 54, no. 1, pp. 80-93, 1983.

[2] P. M. Clarkson and M. J. Hubal, "Exercise-induced muscle damage in humans," American Journal of Physical Medicine and Rehabilitation, vol. 81, no. 11, supplement, pp. S52-S69, 2002.

[3] D. J. Newham, G. McPhail, K. R. Mills, and R. H. T. Edwards, "Ultrastructural changes after concentric and eccentric contractions of human muscle," Journal of the Neurological Sciences, vol. 61, no. 1, pp. 109-122, 1983.

[4] R. W. Ogilvie, R. B. Armstrong, K. E. Baird, and C. L. Bottoms, "Lesions in the rat muscle following eccentrically biased exercise," American Journal of Anatomy, vol. 182, no. 4, pp. 335-346, 1988.

[5] C. D. Balnave and D. G. Allen, "Intracellular calcium and force in single mouse muscle fibres following repeated contractions with stretch," Journal of Physiology, vol. 488, no. 1, pp. 25-36, 1995.

[6] C. T. M. Davies and M. J. White, "Muscle weakness following eccentric work in man," Pflugers Archiv European Journal of Physiology, vol. 392, no. 2, pp. 168-171, 1981.

[7] G. S. Lynch, C. J. Fary, and D. A. Williams, "Quantitative measurement of resting skeletal muscle $\left[\mathrm{Ca}^{2+}\right]$ following acute and long-term downhill running exercise in mice," Cell Calcium, vol. 22, no. 5, pp. 373-383, 1997.

[8] G. L. Warren, D. A. Lowe, and R. B. Armstrong, "Measurement tools used in the study of eccentric contraction-induced injury," Sports Medicine, vol. 27, no. 1, pp. 43-59, 1999.

[9] H. Westerblad and D. G. Allen, "Recent advances in the understanding of skeletal muscle fatigue," Current Opinion in Rheumatology, vol. 14, no. 6, pp. 648-652, 2002.

[10] D. A. Jones, D. J. Newham, and C. Torgan, "Mechanical influences on long-lasting human muscle fatigue and delayedonset pain," Journal of Physiology, vol. 412, pp. 415-427, 1989.

[11] D. J. Newham, K. R. Mills, B. M. Quigley, and R. H. T. Edwards, "Pain and fatigue after concentric and eccentric muscle contractions," Clinical Science, vol. 64, no. 1, pp. 5562, 1983.

[12] E. W. Yeung, J. P. Bourreau, D. G. Allenand, and H. J. Ballard, "Effect of eccentric contraction-induced injury on force and intracellular $\mathrm{pH}$ in rat skeletal muscles," Journal of Applied Physiology, vol. 92, no. 1, pp. 93-99, 2002.

[13] K. K. McCully and J. A. Faulkner, "Injury to skeletal muscle fibers of mice following lengthening contractions," Journal of Applied Physiology, vol. 59, no. 1, pp. 119-126, 1985.

[14] E. A. Abou Salem, N. Fujimaki, and H. Ishikawa, "Formation of unique vacuoles in tenotomized rat soleus muscle fibers," Archives of Histology and Cytology, vol. 64, no. 3, pp. 247-257, 2001.

[15] R. H. T. Edwards, D. K. Hill, D. A. Jones, and P. A. Merton, "Fatigue of long duration in human skeletal muscle after exercise," Journal of Physiology, vol. 272, no. 3, pp. 769-778, 1977.
[16] G. L. Warren, C. P. Ingalls, D. A. Lowe, and R. B. Armstrong, "Excitation-contraction uncoupling: major role in contraction-induced muscle injury," Exercise and Sport Sciences Reviews, vol. 29, no. 2, pp. 82-87, 2001.

[17] G. L. Warren, D. A. Lowe, D. A. Hayes, C. J. Karwoski, B. M. Prior, and R. B. Armstrong, "Excitation failure in eccentric contraction-induced injury of mouse soleus muscle," Journal of Physiology, vol. 468, pp. 487-499, 1993.

[18] H. Westerblad, S. Duty, and D. G. Allen, "Intracellular calcium concentration during low-frequency fatigue in isolated single fibers of mouse skeletal muscle," Journal of Applied Physiology, vol. 75, no. 1, pp. 382-388, 1993.

[19] E. W. Yeung, C. D. Balnave, H. J. Ballard, J. P. Bourreau, and D. G. Allen, "Development of T-tubular vacuoles in eccentrically damaged mouse muscle fibres," Journal of Physiology, vol. 540, no. 2, pp. 581-592, 2002.

[20] R. J. Bloomer, "The role of nutritional supplements in the prevention and treatment of resistance exercise-induced skeletal muscle injury," Sports Medicine, vol. 37, no. 6, pp. 519532, 2007.

[21] M. J. Jackson, M. Khassaf, A. Vasilaki, F. McArdle, and A. McArdle, "Vitamin E and the oxidative stress of exercise," Annals of the New York Academy of Sciences, vol. 1031, pp. 158168, 2004.

[22] J. M. Sacheck and J. B. Blumberg, "Role of vitamin E and oxidative stress in exercise," Nutrition, vol. 17, no. 10, pp. 809814, 2001.

[23] G. W. Burton and K. U. Ingold, "Vitamin $\mathrm{E}$ as an in vitro and in vivo antioxidant," Annals of the New York Academy of Sciences, vol. 570, pp. 7-22, 1989.

[24] A. N. Erin, M. M. Spirin, L. V. Tabidze, and V. E. Kagan, "Formation of $\alpha$-tocopherol complexes with fatty acids. A hypothetical mechanism of stabilization of biomembranes by vitamin E," Biochimica et Biophysica Acta, vol. 774, no. 1, pp. 96-102, 1984.

[25] A. C. Howard, A. K. McNeil, and P. L. McNeil, "Promotion of plasma membrane repair by vitamin e," Nature Communications, vol. 2, no. 1, Article ID 597, 2011.

[26] S. Urano, M. Matsuo, T. Sakanaka et al., "Mobility and molecular orientation of vitamin $\mathrm{E}$ in liposomal membranes as determined by $19 \mathrm{~F} \mathrm{NMR}$ and fluorescence polarization techniques," Archives of Biochemistry and Biophysics, vol. 303, no. 1, pp. 10-14, 1993.

[27] D. M. Bailey, C. Williams, J. A. Betts, D. Thompson, and T. L. Hurst, "Oxidative stress, inflammation and recovery of muscle function after damaging exercise: effect of 6-week mixed antioxidant supplementation," European Journal of Applied Physiology, vol. 111, no. 6, pp. 925-936, 2011.

[28] L. J. Beaton, D. A. Allan, M. A. Tarnopolsky, P. M. Tiidus, and S. M. Phillips, "Contraction-induced muscle damage is unaffected by vitamin E supplementation," Medicine and Science in Sports and Exercise, vol. 34, no. 5, pp. 798-805, 2002.

[29] R. J. Bloomer, A. H. Goldfarb, M. J. McKenzie, T. You, and L. Nguyen, "Effects of antioxidant therapy in women exposed to eccentric exercise," International Journal of Sport Nutrition and Exercise Metabolism, vol. 14, no. 4, pp. 377-388, 2004.

[30] P. Jakeman and S. Maxwell, "Effect of antioxidant vitamin supplementation on muscle function after eccentric exercise," European Journal of Applied Physiology and Occupational Physiology, vol. 67, no. 5, pp. 426-430, 1993.

[31] A. A. Theodorou, M. G. Nikolaidis, V. Paschalis et al., "No effect of antioxidant supplementation on muscle performance and blood redox status adaptations to eccentric training," 
American Journal of Clinical Nutrition, vol. 93, no. 6, pp. 13731383, 2011.

[32] A. Kyparos, S. Sotiriadou, V. Mougios et al., "Effect of 5-day vitamin E supplementation on muscle injury after downhill running in rats," European Journal of Applied Physiology, vol. 111, no. 10, pp. 2557-2569, 2011.

[33] J. H. Van Der Meulen, A. McArdle, M. J. Jackson, and J. A. Faulkner, "Contraction-induced injury to the extensor digitorum longus muscles of rats: the role of vitamin E," Journal of Applied Physiology, vol. 83, no. 3, pp. 817-823, 1997.

[34] J. A. Warren, R. R. Jenkins, L. Packer, E. H. Witt, and R. B. Armstrong, "Elevated muscle vitamin E does not attenuate eccentric exercise-induced muscle injury," Journal of Applied Physiology, vol. 72, no. 6, pp. 2168-2175, 1992.

[35] A. Shafat, P. Butler, R. L. Jensen, and A. E. Donnelly, "Effects of dietary supplementation with vitamins $\mathrm{C}$ and $\mathrm{E}$ on muscle function during and after eccentric contractions in humans," European Journal of Applied Physiology, vol. 93, no. 1-2, pp. 196-202, 2004.

[36] F. Eusebi, R. Miledi, and T. Takahashi, "Calcium transients in mammalian muscles," Nature, vol. 284, no. 5756, pp. 560-561, 1980.

[37] D. A. Jones, "High- and low-frequency fatigue revisited," Acta Physiologica Scandinavica, vol. 156, no. 3, pp. 265-270, 1996.

[38] R. B. Armstrong, "Initial events in exercise-induced muscular injury," Medicine and Science in Sports and Exercise, vol. 22, no. 4, pp. 429-435, 1990.

[39] J. Hidalgo, L. Campmany, M. Borras, J. S. Garvey, and A. Armario, "Metallothionein response to stress in rats: role in free radical scavenging," American Journal of Physiology, vol. 255, no. 4, part 1, pp. E518-E524, 1988.

[40] S. Kovacheva and S. R. Ribarov, "Lipid peroxidation in lung of rat stressed by immobilization: effects of vitamin $\mathrm{E}$ supplementation," Lung, vol. 173, no. 4, pp. 255-263, 1995.

[41] S. Sotiriadou, A. Kyparos, M. Albani et al., "Soleus muscle force following downhill running in ovariectomized rats treated with estrogen," Applied Physiology, Nutrition and Metabolism, vol. 31, no. 4, pp. 449-459, 2006.

[42] H. Takekura, N. Fujinami, T. Nishizawa, H. Ogasawara, and N. Kasuga, "Eccentric exercise-induced morphological changes in the membrane systems involved in excitation-contraction coupling in rat skeletal muscle," Journal of Physiology, vol. 533, no. 2, pp. 571-583, 2001.

[43] D. W. Nierenberg and S. L. Nann, "A method for determining concentrations of retinol, tocopherol, and five carotenoids in human plasma and tissue samples," American Journal of Clinical Nutrition, vol. 56, no. 2, pp. 417-426, 1992.

[44] J. Cohen, Statistical Power Analysis for the Behavioral Sciences, Lawrence Erlbaum, Hillsdale, NJ, USA, 1998.

[45] A. Kyparos, C. Matziari, M. Albani, G. Arsos, S. Sotiriadou, and A. Deligiannis, "A decrease in soleus muscle force generation in rats after downhill running," Canadian Journal of Applied Physiology, vol. 26, no. 4, pp. 323-335, 2001.

[46] C. P. Ingalls, G. L. Warren, J. H. Williams, C. W. Ward, and R. B. Armstrong, "E-C coupling failure in mouse EDL muscle after in vivo eccentric contractions," Journal of Applied Physiology, vol. 85, no. 1, pp. 58-67, 1998.

[47] J. L. Vergara, S. I. Rapoport, and V. Nassar Gentina, "Fatigue and posttetanic potentiation in single muscle fibers of the frog," American Journal of Physiology, vol. 232, no. 5, pp. C185C190, 1977.

[48] H. Westerblad and D. G. Allen, "Changes of myoplasmic calcium concentration during fatigue in single mouse muscle fibers," Journal of General Physiology, vol. 98, no. 3, pp. 615635, 1991.

[49] D. A. Essig and T. M. Nosek, "Muscle fatigue and induction of stress protein genes: a dual function of reactive oxygen species?" Canadian Journal of Applied Physiology, vol. 22, no. 5, pp. 409-428, 1997.

[50] G. D. Lamb and H. Westerblad, "Acute effects of reactive oxygen and nitrogen species on the contractile function of skeletal muscle," Journal of Physiology, vol. 589, no. 9, pp. 2119-2127, 2011.

[51] H. Westerblad and D. G. Allen, "Emerging roles of ROS/RNS in muscle function and fatigue," Antioxidants and Redox Signaling, vol. 15, no. 9, pp. 2487-2499, 2011.

[52] G. D. Lamb, "Excitation-contraction coupling and fatigue mechanisms in skeletal muscle: studies with mechanically skinned fibres," Journal of Muscle Research and Cell Motility, vol. 23, no. 1, pp. 81-91, 2002.

[53] G. D. Lamb, "Mechanisms of excitation-contraction uncoupling relevant to activity-induced muscle fatigue," Applied Physiology, Nutrition and Metabolism, vol. 34, no. 3, pp. 368372,2009

[54] T. You, A. H. Goldfarb, R. J. Bloomer, L. Nguyen, X. Sha, and M. J. McKenzie, "Oxidative stress response in normal and antioxidant supplemented rats to a downhill run: changes in blood and skeletal muscles," Canadian Journal of Applied Physiology, vol. 30, no. 6, pp. 677-689, 2005.

[55] M. Meydani, W. J. Evans, G. Handelman et al., "Protective effect of vitamin $\mathrm{E}$ on exercise-induced oxidative damage in young and older adults," American Journal of Physiology, vol. 264, no. 5, pp. R992-R998, 1993.

[56] M. G. Nikolaidis, A. Kyparos, C. Spanou, V. Paschalis, A. A. Theodorou, and I. S. Vrabas, "Redox biology of exercise: an integrative and comparative consideration of some overlooked issues," The Journal of Experimental Biology, vol. 215, no. 10, pp. 1615-1625, 2012. 


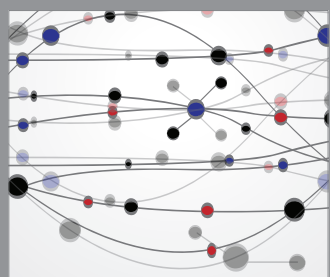

The Scientific World Journal
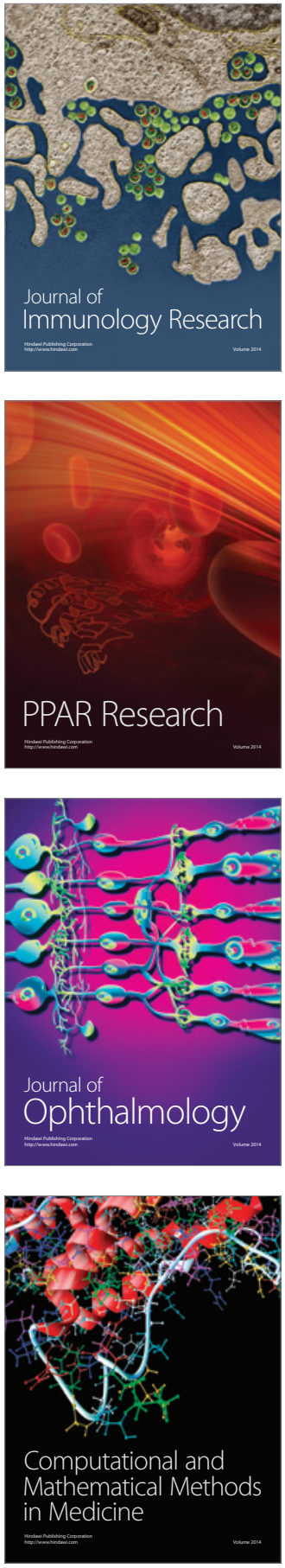

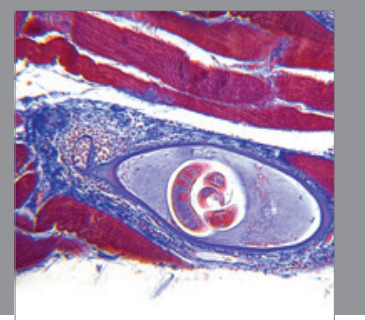

Gastroenterology

Research and Practice
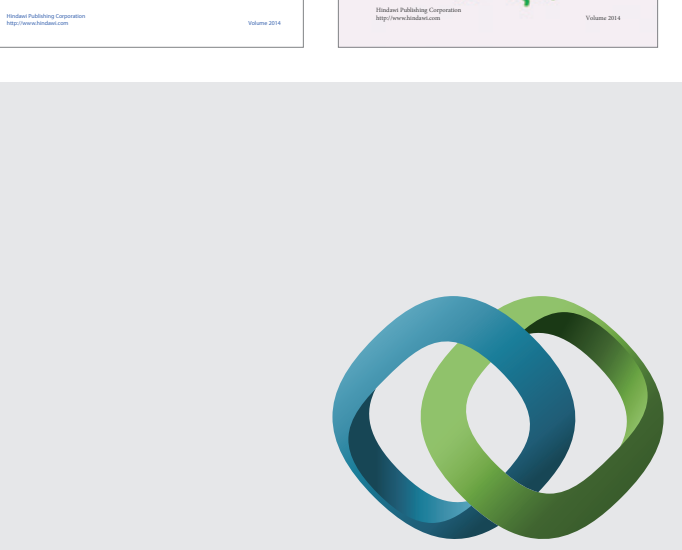

\section{Hindawi}

Submit your manuscripts at

http://www.hindawi.com
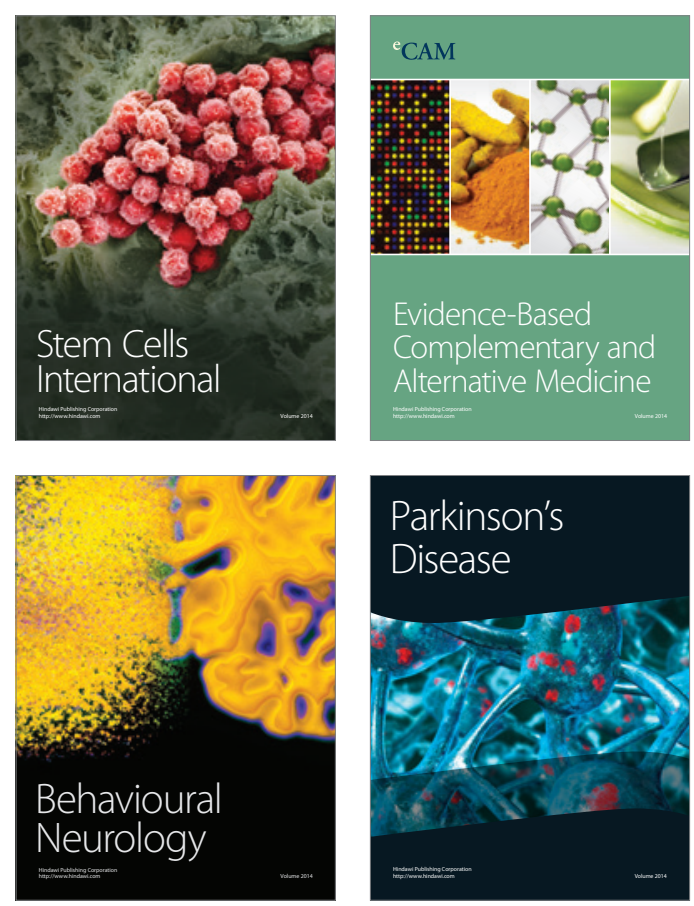

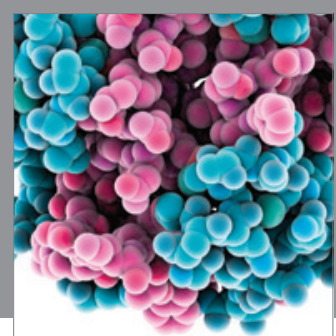

Journal of
Diabetes Research

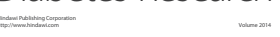

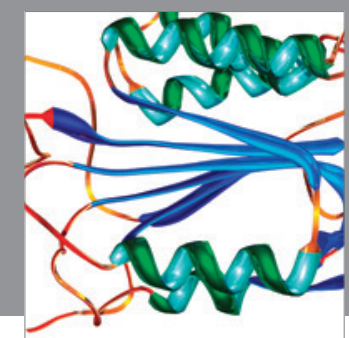

Disease Markers
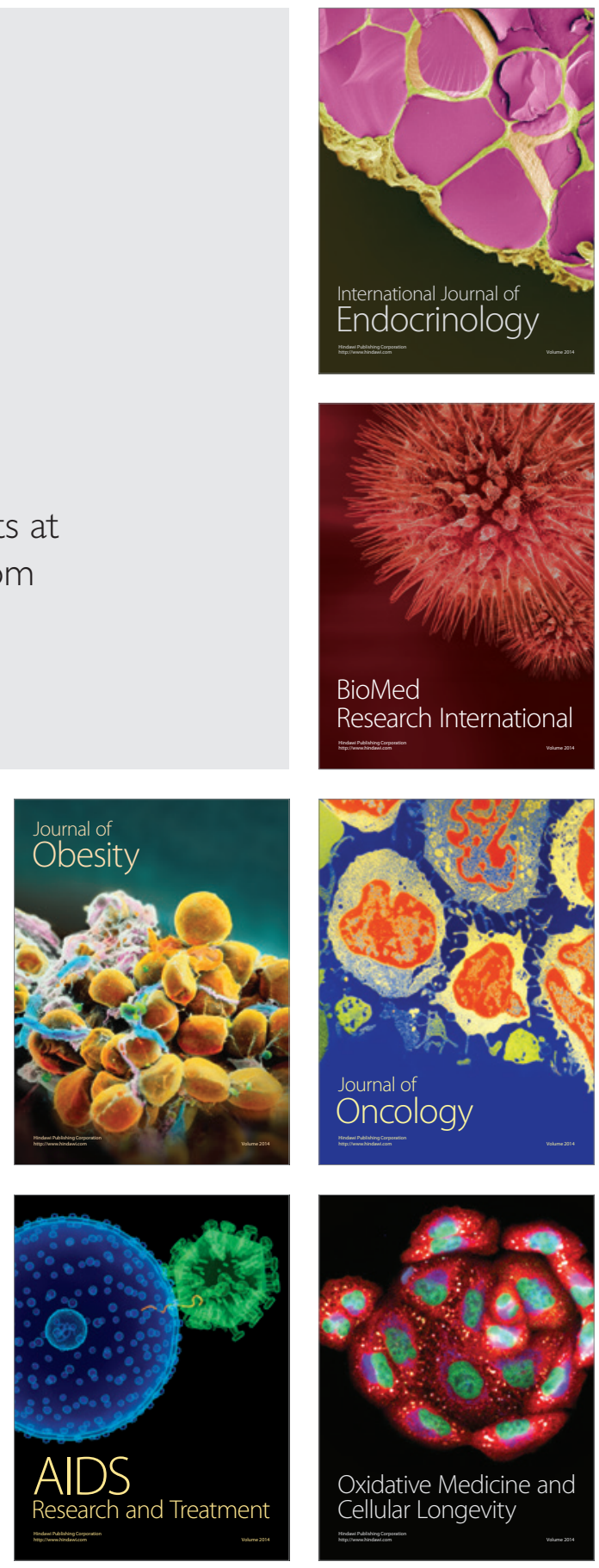